\title{
Outcomes in Patients with First Episode of Optic Neuritis
}

\author{
Ebin Jose $^{1}$, Srikumar B ${ }^{2^{*}}$, Ram Mohan ${ }^{3}$
}

${ }^{1}$ Senior Resident, Department of Neurology, Government Medical College Thiruvananthapuram, Kerala 695011, India

${ }^{2}$ Professor, Department of Neurology, Government Medical College Thiruvananthapuram, Kerala 695011, India

${ }^{3}$ Associate Professor, Department of Neurology, Government Medical College Thiruvananthapuram, Kerala 695011, India

DOI: $10.36347 /$ sjams.2020.v08i11.042

| Received: 01.10.2020 | Accepted: 17.10.2020 | Published: 29.11.2020

*Corresponding author: Srikumar B

Abstract

Background: Optic neuritis (ON) is characterized by unilateral or bilateral sudden loss of vision, often accompanied by periocular pain due to idiopathic, inflammatory, infectious or demyelinating causes. When compared to the Western population, various studies from Asia suggest that there is difference in aetiology, clinical presentation and prognosis of optic neuritis. Few Indian studies are available evaluating the natural course following first attack of optic neuritis. Methods: This prospective observational study was conducted on 20 patients. Inclusion criteria included age range of 18 to 50 years, acute unilateral or bilateral optic neuritis with visual symptoms for less than or equal to 1 week associated with at least 2 of the following: colour vision abnormality, pain on eye movement, afferent papillary defect, and abnormal visual-evoked potential (VEP). Patients having toxic, metabolic, vascular, and infective causes for optic neuritis were excluded from the study. CSF study including oligo-clonal band (OCB), S. NMO antibody, MRI Brain and spine were done for all patients. Patients were followed up at 3 months, 6 months and 1 year with detailed clinical examination and VEP to assess visual recovery and development of new neurological deficits. Results: Female $(n=12)$ preponderance was seen in a ratio of 3: 2. Recurrent optic neuritis was seen in one patient with idiopathic optic neuritis. During one year follow up, 3 patients developed additional neurological deficits of which 2 were having MS and 1 having NMO. Patients with MS were having short segment demyelinating lesion and the one with NMO was having LETM and cord oedema. Bilateral ON was seen in 2 patients (one S. NMO and the other S. anti MOG antibody positive). There was statistically significant association with (p value $=0.01$ ) increase in the number of lesions in the follow up MRI brain and development of multiple sclerosis. Visual outcome was better in patients with idiopathic ON and optic neuritis associated with MS. Conclusion: In patients presenting with optic neuritis, those with NMO were having the worst visual outcome at initial presentation and during one year follow up. There is statistically significant association between risk of development of multiple sclerosis and increase in the number of lesions during follow up MRI brain.

Keywords: Optic Neuritis Treatment Trial, Multiple sclerosis, Neuromyelitis optica, anti MOG antibody.

Copyright $\odot 2020$ The Author(s): This is an open-access article distributed under the terms of the Creative Commons Attribution 4.0 International License (CC BY-NC 4.0) which permits unrestricted use, distribution, and reproduction in any medium for non-commercial use provided the original author and source are credited.

\section{INTRODUCTION}

Optic neuritis is an inflammatory, demyelinating condition of the optic nerve characterised by acute, usually monocular, visual loss [1]. Majority of the acute demyelinating optic neuritis occurs in women, with a peak incidence in the age group of 20-40 years [2]. Optic neuritis patients usually present with subacute unilateral loss of vision, pain during eye movements and impaired colour vision. Symptoms usually worsen over a period of a few days to 2 weeks but spontaneously recover in more than $90 \%$ of the cases. Most of the cases of optic neuritis (ON) are idiopathic in origin. This can occur as an isolated syndrome or as an initial manifestation of multiple sclerosis (MS). Optic neuritis can be the presenting symptom of MS in $25 \%$ of cases. In about $70 \%$ of the cases optic neuritis can occur during the disease course, usually in the relapsing-remitting phase. Studies indicate that $34-75 \%$ of patients presenting with optic neuritis convert to clinically definite MS [3]. When optic neuritis occurs, brain magnetic resonance imaging (MRI) often demonstrates white-matter T2 signal abnormalities consistent with demyelination [4]. Approximately 50\% of patients with isolated ON develop definite MS within 15 years. An asymptomatic demyelinating lesion in the central nervous system (CNS) is the most important predictor for subsequent development of multiple sclerosis after an episode of optic neuritis. Neuromyelitis optica (NMO) is an autoimmune demyelinating disorder of the central nervous system that preferentially affects the optic nerves and spinal cord. Isolated optic neuritis $(\mathrm{ON})$ can be the presenting 
symptom in NMO [5]. NMO accounts for 20-48\% of demyelinating lesions in Asian population. About fifty percentage of NMO patients present with isolated optic neuritis, of which nearly $20 \%$ is bilateral [6].

Objective of The Study: To study outcomes in patients presenting with first episode of optic neuritis.

\section{Materials and Methods \\ Study Design: Longitudinal study Setting}

Neurology Department, Government Medical College, Thiruvananthapuram. The study started in April 2017 and completed in March 2018.

\section{Study Population}

Those patients attending outpatient department or admitted in neurology ward whose symptoms were consistent with the diagnosis of optic neuritis, satisfying inclusion criteria.

\section{Operational definition}

Optic neuritis was defined by history of unilateral or bilateral visual loss of acute onset of 1 week or less in duration, associated with at least 2 of the following criteria: colour vision abnormality, pain on eye movement, afferent papillary defect, and abnormal visual-evoked potential. Outcomes were assessed at 3 months, 6 months and 1 year and included, improvement in visual acuity, colour vision, afferent pupillary defect and development of new neurological deficits.

\section{Inclusion Criteria}

1. Age range of 18 to 50 years

2. Acute unilateral or bilateral optic neuritis with visual symptoms for 1 week associated with at least 2 of the following: colour vision abnormality, pain on eye movement, afferent papillary defect, and abnormal visual-evoked potential (VEP).

\section{Exclusion Criteria}

1. Patients having toxic, metabolic, vascular, and infective causes for optic neuritis

2. Patients with past history of optic neuritis and already diagnosed cases of multiple sclerosis or neuromyelitis optica.

\section{Sample Size}

The average number of patients with a diagnosis of first episode of acute optic neuritis attending neurology OPD or wards per year was 20 . So the study population included a total of 20 subjects meeting inclusion criteria.

Sampling Technique: Consecutive sampling.

\section{Methodology}

Patients with optic neuritis were included in the study after obtaining informed consent. Optic neuritis was diagnosed on the basis of history and clinical examination, which include sudden unilateral or bilateral visual loss of less than 1 week duration, presence of relative afferent pupillary defect, dyschromatopsia, and normal or swollen optic disc on fundus examination. Detailed history was obtained, which included onset of visual loss, duration, association with pain, any previous attack, and history of any other neurological symptoms. Clinical examination included visual acuity (VA), field of vision, evaluation of pupils, and fundus examination. All patients had undergone detailed neurological evaluation to look for any focal neurological deficits. All patients' haemogram, peripheral smear, human immunodeficiency virus (HIV) test, VDRL, anti-nuclear antibody (ANA), anti-double stranded DNA antibody (dsDNA) and chest X-ray were done. CSF study including oligo-clonal band (OCB) was done for all patients. S. NMO (AQP4-IgG) antibody testing using cell based assay was done for all patients. MRI Brain plain and contrast was done for all patients to look for any contrast enhancement of optic nerve, T2/FLAIR white matter hyper-intense lesions, contrast enhancement of these lesions. Multiple sclerosis was diagnosed using the 2010 Modified McDonald criteria and NMOSD by the 2015 international consensus diagnostic criteria for NMOSD [7, 8]. According to the Modified McDonald criteria, at least one lesion must occur in two out of four typical locations to satisfy MRI criteria for dissemination in space periventricular, juxtacortical, infratentorial and spinal cord. For the dissemination in time, there should be either simultaneous presence of asymptomatic gadoliniumenhancing and non-enhancing lesions at any time, or a new T2 or T1 gadolinium-enhancing lesion on followup MRI scan, irrespective of the timing of the initial scan. Visual evoked response, and colour vision with Ishihara pseudo isochromatic plates was done for all patients. Patients were followed up at 3 months, 6 months and 1 year with detailed clinical examination and VEP to assess visual recovery and development of new neurological deficits.

\section{Data Collection and Analysis}

Data was collected using a predesigned proforma and this was entered into Microsoft Excel spread sheet. Statistical analysis was performed using SSPS version 18.

\section{RESULTS}

The mean age of study population was $26.3 \pm 6.54$ years (range $17 \quad 38$ years). Female preponderance was seen with a ratio of $3: 2$ (male $40 \%$ $(n=8)$, female $60 \%(n=8)$. The mean duration of illness at the time of initial presentation was $5.35 \pm 1.35$ days (range 3- 7 days). The etiology of optic neuritis is shown in Figure-1. 


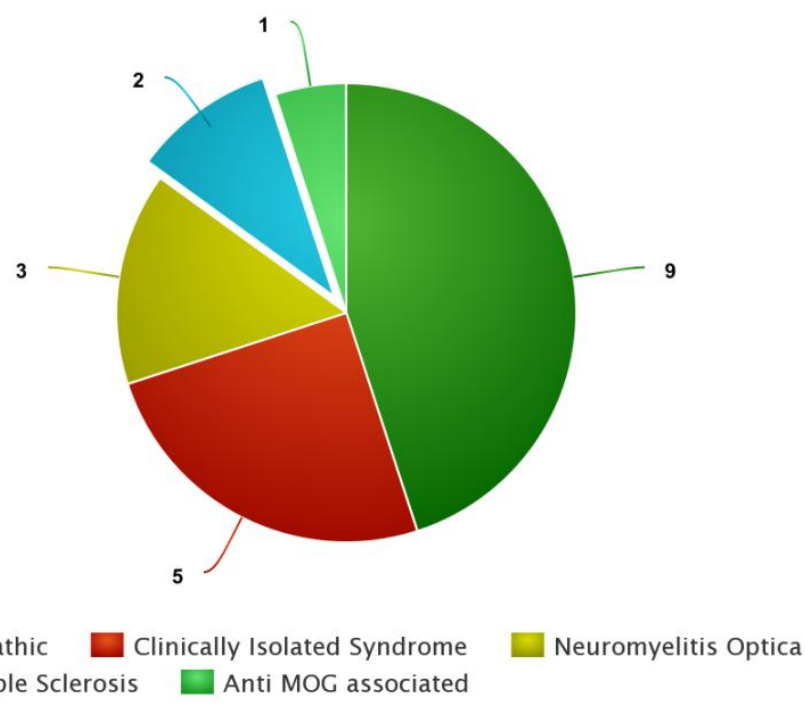

Fig-1: Pie chart showing frequency of etiology of optic neuritis cases

\section{Visual Acuity}

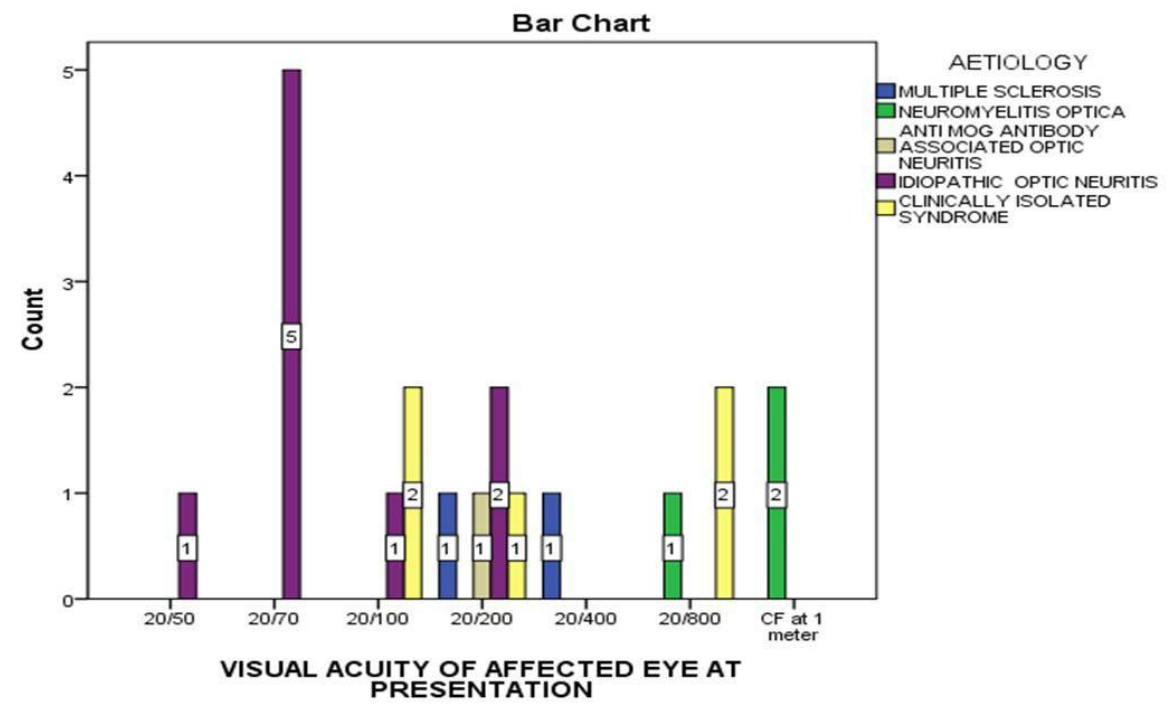

Fig-2: Visual acuity of affected eye at initial presentation according to aetiology

Patients with NMO had the worst best corrected visual acuity at initial presentation. Two patients with $\mathrm{NMO}$ had a visual acuity of counting finger at 1 meter and one had a visual acuity of 20/800. 


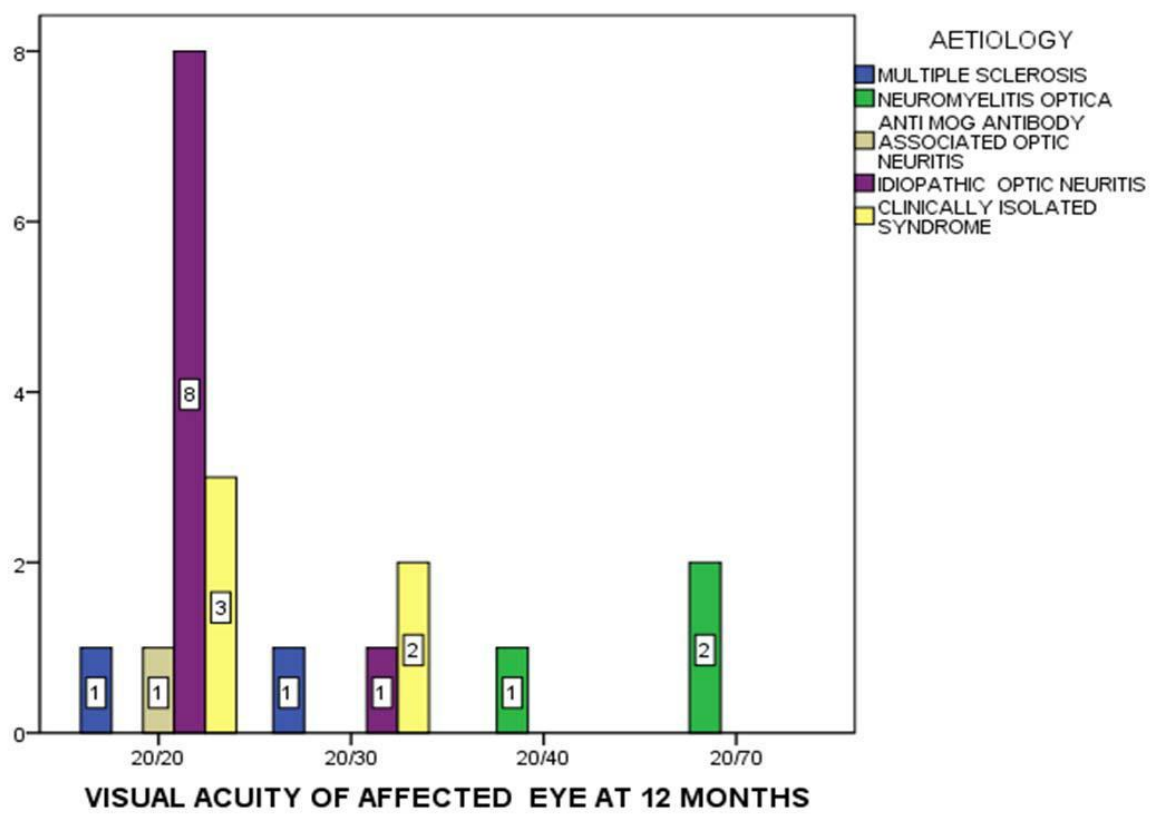

Fig-3: Visual of affected eye at 1 year according to aetiology

At 1 year, 13 patients $(65 \%)$ had a normal visual acuity of 20/20. Patients with NMO had the worst visual outcome. Two out of the 3 patients with NMO had a visual acuity of 20/70 and 1 patient had a visual acuity of 20/40. One patient with anti MOG antibody had good recovery of vision with a visual acuity of 20/20 at one year. CSF was normal in 19 patients and 1 patient with anti MOG antibody seropositivity had lymphocytic pleocytosis.

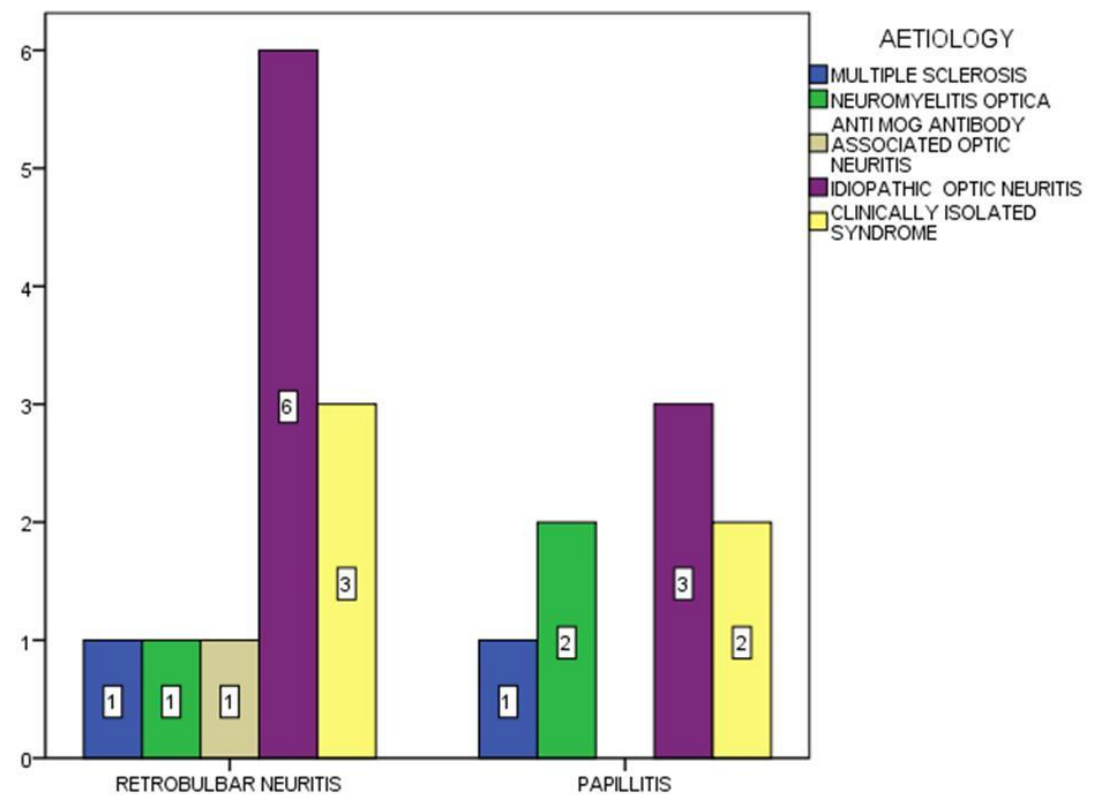

Fig-4: Fundus According to Aetiology

Retrobulbar neuritis (RBN) was seen in 12 patients $(60 \%)$ and papillitis in 8 patients $(40 \%)$. 


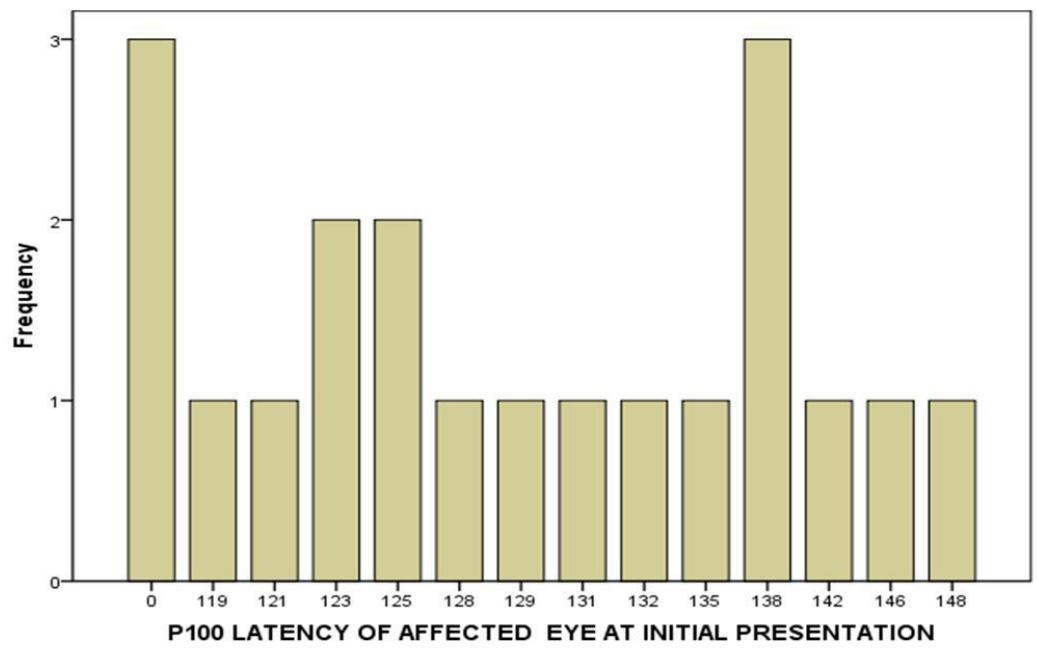

Fig-5: VEP P100 LATENCY AT INITIAL PRESENTATION

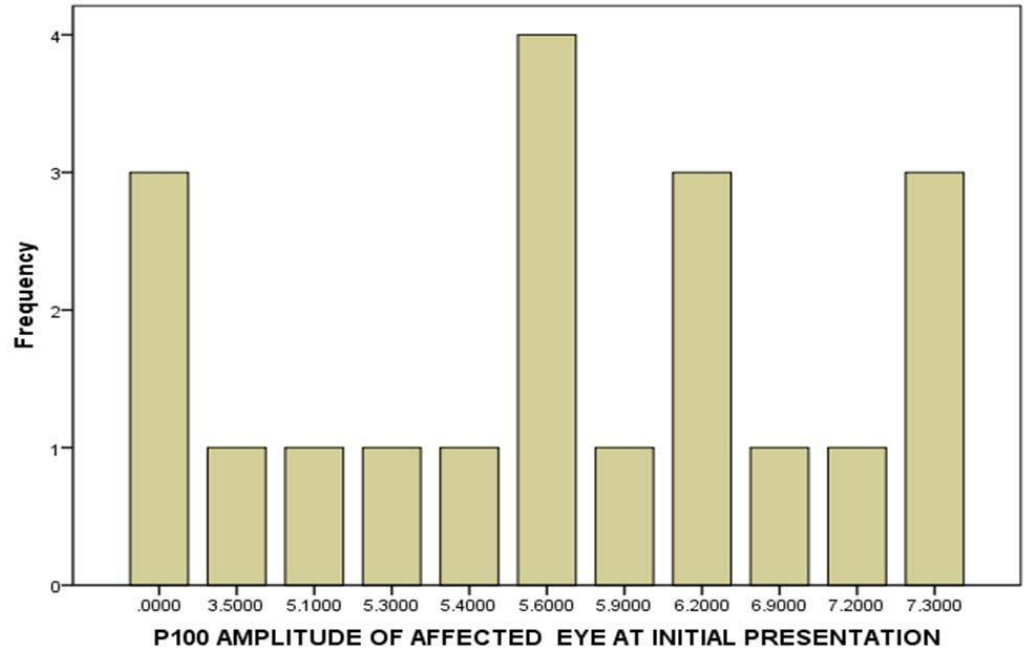

Fig-6: VEP P100 AMPLITUDE AT INITIAL PRESENTATION

The median P100 latency at initial presentation was $128.5 \mathrm{~ms}$ and median P100 amplitude was 5.6 microvolt. There were no elicitable wave forms in all the three patients with NMO.

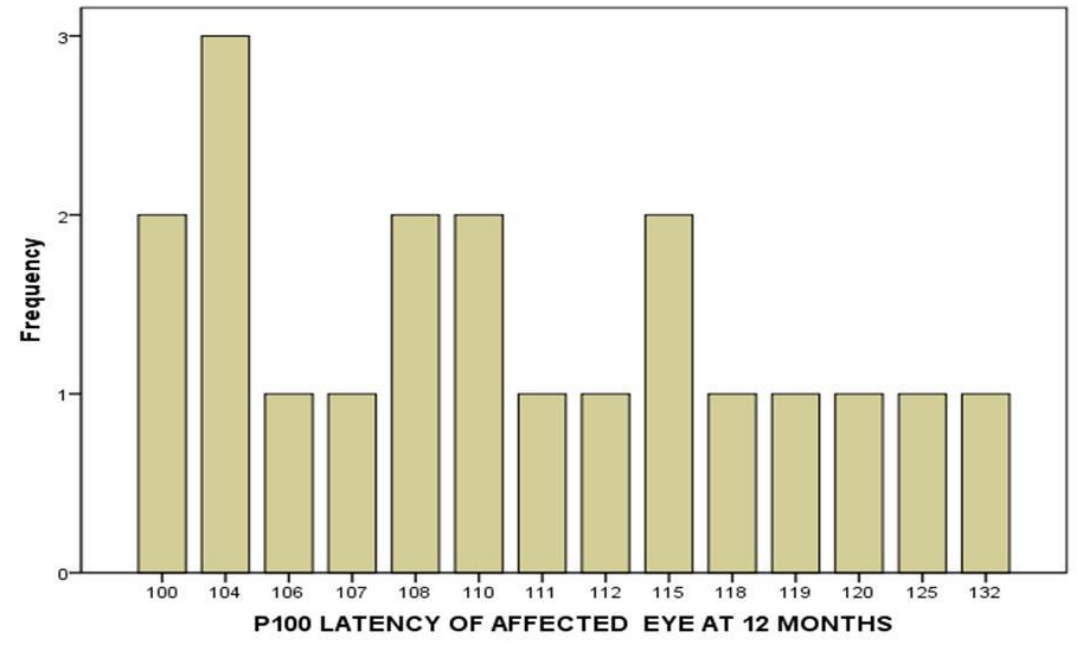

Fig-7: Vep P100 Latency at 1 Year 


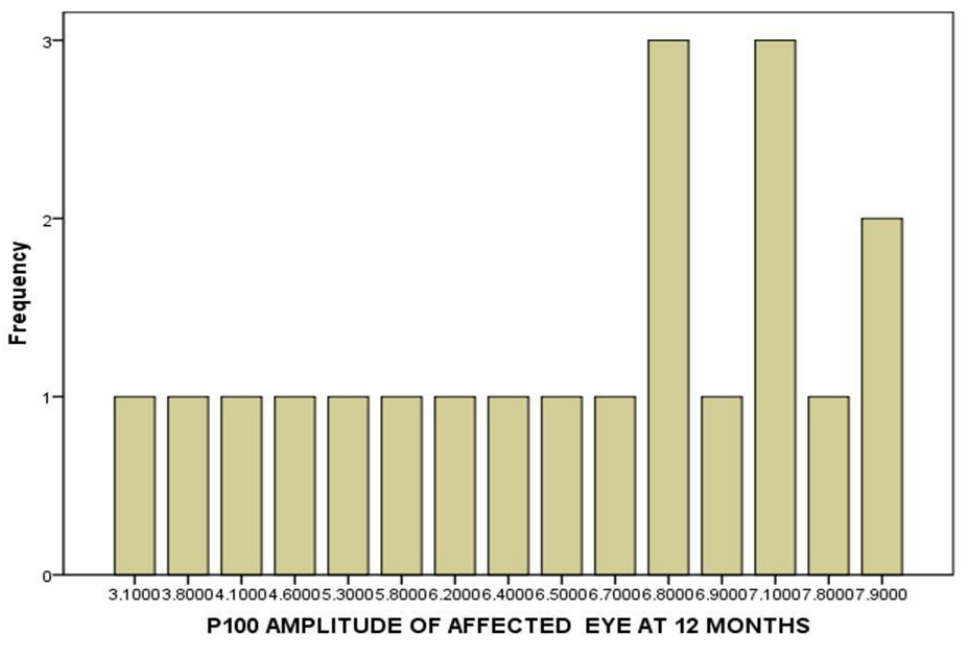

Fig-8: VEP P100 AMPLITUDE AT 1 YEAR

The median P100 latency at initial presentation was $111.4 \mathrm{~ms}$ and median P100 amplitude was 6.75 microvolt. All the 3 patients with NMO and 1 patient with MS were having P100 amplitude less than 5 microvolt. P100 latency was prolonged in 2 patients with MS, 4 patients with CIS, and one patient with idiopathic optic neuritis.

Table-1: Serum NMO antibody and CSF OCB

\begin{tabular}{|c|c|c|c|}
\hline & Present & Absent & Total \\
\hline $\begin{array}{l}\text { Serum } \\
\text { Antibody }\end{array}$ & 3 & 17 & 20 \\
\hline CSF OCB & 4 & 16 & 20 \\
\hline
\end{tabular}

S. NMO was positive in 3 patients $(15 \%)$ and CSF OCB was positive in 6 patients $(30 \%)$. One patient was positive for serum anti MOG antibody. One patient with NMO and one patient with anti MOG antibody presented with bilateral optic neuritis.

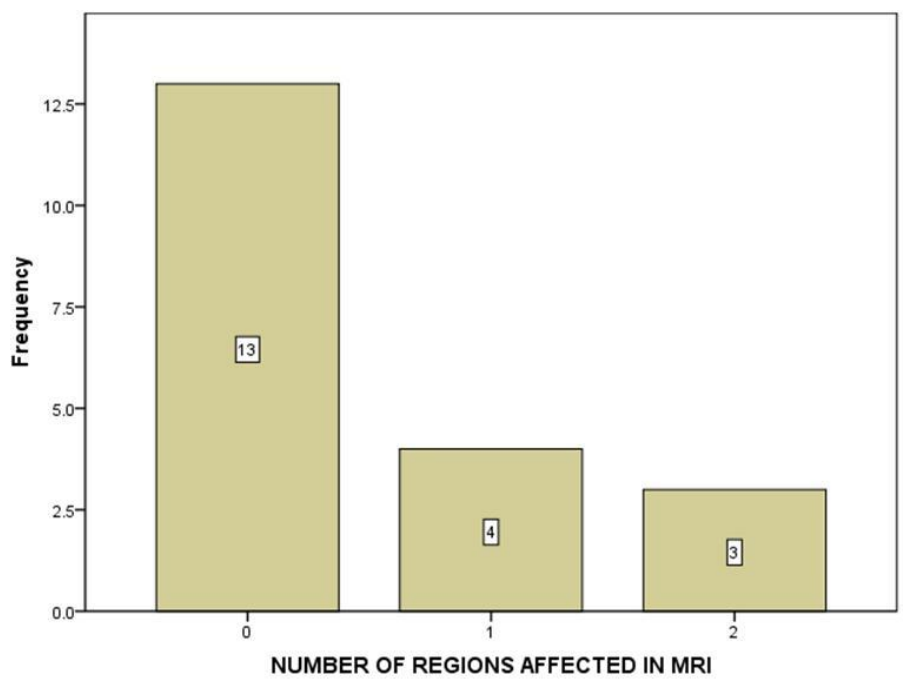

Fig-9: Number of Regions Involved in MRI Brain

Initial MRI brain was abnormal in 7 patients (35\%), which was showing T2/ FLAIR hyper intense lesions in juxtacortical, periventricular or infratentorial region. Of these 2 patients had a relapse within one year and eventually diagnosed to have definite MS. MRI orbit was abnormal in 12 patients $(60 \%)$, showing T2/ FLAIR hyper intense lesions and enhancement of the optic nerve. MRI orbit was abnormal all patients diagnosed to have multiple sclerosis $(n=2)$, clinically isolated syndrome $(\mathrm{n}=5)$, and neuromyelitis optica $(n=3)$. One patient with anti MOG antibody was having normal MRI brain and orbit. Initial MRI cervical spine was normal in all patients. 
Quadripare sis: 1

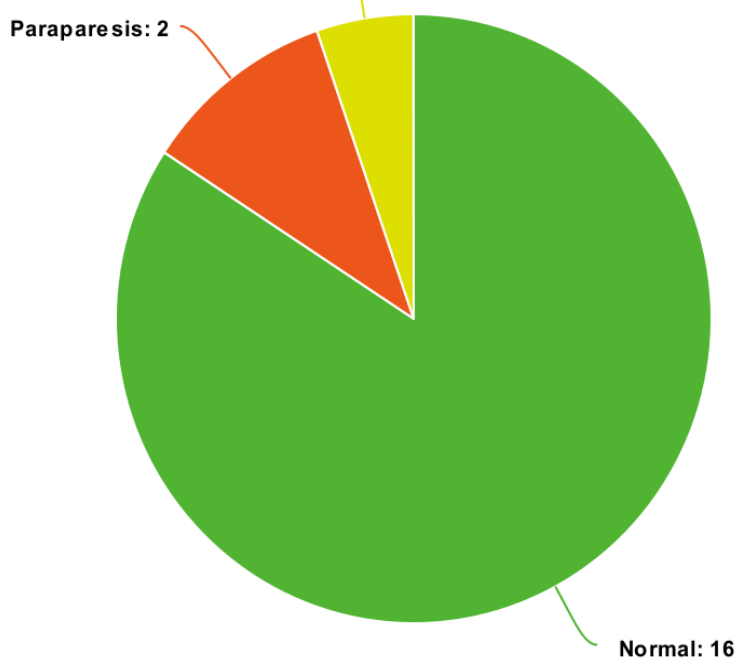

Fig-10: Pie char showing frequency of neurological Deficits at one year follow-up
One patient with idiopathic optic neuritis had a relapse of optic neuritis. Two patients with a diagnosis of CIS developed additional deficits during follow up and converted into definite MS. One patient with MS developed quadriparesis within one year and MRI cervical spine showed short segment demyelination lesion. Another patient with MS developed paraparesis and MRI thoracic spine showed demyelinating lesion. One patient with NMO developed paraparesis and MRI thoracic spine showed long segment demyelinating lesion (LETM).

\section{Treatment}

In one patient with MS and one patient with CIS, immunomodulatory therapy with interferon beta 1a was started. One patient with MS did not opt for the same due to financial constraints. Two patients with NMO were started on azathioprine. One patient with NMO who had a relapse was treated with intravenous methylprednisolone followed by injection rituximab once monthly.

Table-2: Univariate Analysis of Risk Factors for Development of New Symptoms

\begin{tabular}{|l|l|l|l|l|}
\hline RISK FACTORS & & Odds & \multicolumn{2}{l|}{ 95\% C.I } \\
\hline & P value & \multicolumn{1}{|c|}{ Ratio } & Lower & Upper \\
\hline Age & .289 & 1.177 & .871 & 1.590 \\
\hline Visual acuity of affected eye at presentation & .964 & .958 & .148 & 6.222 \\
\hline Regions affected in MRI & .443 & .010 & .000 & 1299.004 \\
\hline P100 latency of affected eye at initial presentation & .593 & .970 & .870 & 1.083 \\
\hline P100 amplitude of affected eye at initial presentation & .949 & 1.078 & .112 & 10.382 \\
\hline
\end{tabular}

Univariate logistic regression was used for continuous variables like age, visual acuity of affected eye at presentation, regions affected in initial MRI brain, P100 latency and amplitude at initial presentation as risk factor for the development of new symptoms and none were found to be statistically significant.

Table-3: Relationship of MRI and CSF OCB with development of new symptoms during follow up

\begin{tabular}{|c|c|c|c|}
\hline \multirow[t]{2}{*}{ NEW SYMPTOMS DURING FOLLOW UP } & \multicolumn{2}{|c|}{ MRI BRAIN } & \multirow[b]{2}{*}{ Total } \\
\hline & NORMAL & ABNORMAL & \\
\hline $\mathrm{NO}$ & 11 & 5 & 16 \\
\hline \multirow[t]{3}{*}{ YES } & 2 & 2 & 4 \\
\hline & \multicolumn{2}{|c|}{ MRI ORBIT } & \\
\hline & NORMAL & ABNORMAL & Total \\
\hline $\mathrm{NO}$ & 8 & 8 & 16 \\
\hline \multirow[t]{3}{*}{ YES } & 0 & 4 & 4 \\
\hline & \multicolumn{2}{|c|}{ CSF OCB } & \\
\hline & ABSENT & PRESENT & Total \\
\hline $\mathrm{NO}$ & 8 & 8 & 16 \\
\hline YES & 0 & 4 & 4 \\
\hline
\end{tabular}

Risk for development of new symptoms and initial MRI brain was assessed using Chi square test. 2 out of the 7 patients with an abnormal MRI brain developed new symptoms, but it was not statistically significant $(\mathrm{p}=0.6)$.
Risk for development of new symptoms and initial MRI orbit was assessed using Chi square test. 4 out of the 12 patients with an abnormal MRI orbit developed new symptoms, which was statistically significant $(p=0.05)$. 
Table-4: New symptoms during follow up and CSF OCB

\begin{tabular}{|l|l|l|l|}
\hline & \multicolumn{2}{|l|}{ NEW SYMPTOMS DURING } & \\
\hline & \multicolumn{1}{|c|}{ NOLLOW UP } & YES & Total \\
\hline & \multicolumn{1}{|c|}{ NO } & 2 & 14 \\
\hline CSF OCB ABSENT & 12 & 2 & 6 \\
\hline PRESENT & 4 & 4 & 20 \\
\hline Total & 16 & \multicolumn{2}{l}{} \\
\hline
\end{tabular}

Risk for development of new symptoms and CSF OCB was assessed using Chi square test. 2 out of the 6 patients with CSF OCB developed new symptoms, which was not statistically significant $(\mathrm{p}=$ $0.59)$.

Table-5: New symptoms during follow up and S. NMO antibody

\begin{tabular}{|l|l|l|l|l|}
\hline & \multicolumn{3}{|c|}{ S. NMO ANTIBODY } & \multicolumn{1}{|c|}{ Total } \\
\hline & & \multicolumn{1}{|c|}{ ABSENT } & PRESENT & \multicolumn{1}{|c|}{ NRE } \\
\hline NEW SYMPTOMS & NO & 14 & 2 & 16 \\
\cline { 2 - 5 } DURING FOLLOW UP & YES & 3 & 1 & 4 \\
\hline Total & & 17 & 3 & 20 \\
\hline
\end{tabular}

Risk for development of new symptoms and CSF OCB was assessed using Chi square test 1 out of 3 patients with positive S. NMO antibody developed new symptoms, which was not statistically significant ( $\mathrm{p}$ $=0.5$ ).

\section{DiscUSSION}

This prospective observational study was conducted in the Department of Neurology, Government Medical College, Thiruvananthapuram over a period of one year to study outcomes in patients presenting with first episode of optic neuritis. The mean age of study population was $26.3 \pm 6.54$ years. Female preponderance was seen in the present study, similar to that of ONTT. ${ }^{2}$ Pain during eye movements was observed in fourteen $(70 \%)$ patients. In the ONTT, ocular pain was reported in $92 \%$ of the cases. In the study by Saxena et al., pain during eye movements was seen in $73.4 \%$ of the cases [9]. This finding was consistent with observations made in studies from other Asian countries [10].

In this study patients with NMO had the least corrected visual acuity during an acute attack. Of the 3 patients with NMO, 2 had a visual acuity of counting finger at 1 meter and one had a visual acuity of 20/800, which was consistent with study by Wingerchuk et al., that during an acute attack, more than $80 \%$ of patients with NMO experience severe loss of visual acuity (< 20/200) in contrast to $36 \%$ in patients with MS [6]. At 1 year, compared to others, patients with NMO had guarded visual prognosis, which was in accordance with observation made in the study by Merle et al., [11] In the present study two out of the 3 patients with NMO had a visual acuity of $20 / 70$ and 1 patient had a visual acuity of 20/40.

There was prolongation of P100 latency in all the cases, except those with NMO, at initial presentation with a median value of 128.5 millisecond and P100 amplitude median value of 5.6 microvolt.
There were no elicitable wave forms in all the three patients with NMO. During one year follow up, all the 3 patients with NMO and 1 patient with MS were having P100 amplitude less than 5 microvolt. P100 latency was prolonged in 2 patients with MS, 4 patients with CIS, and one patient with idiopathic optic neuritis, which indicates that the severity of optic nerve damage is more in patients with neuromyelitis optica.

During one year follow up, 3 patients developed additional neurological deficits of which 2 were having MS and 1 having NMO. Patients with MS were having short segment demyelinating lesion and the one with NMO was having LETM and cord oedema, in accordance with observations made in study by Kiyat Atamer et al., [12].

There was statistically significant association with $(\mathrm{p}$ value $=0.01)$ increase in the number of lesions in the follow up MRI brain and development of multiple sclerosis. In the ONTT, in patients with one or more lesions on initial MRI, the risk of MS after 15 years was 72 percent [13]. In accordance with the ONTT, there was no significant difference in the risk between those with single versus multiple lesions, in the initial MRI. There was no statistically significant association between visual acuity of affected eye at presentation, regions affected in initial MRI brain, P100 latency and amplitude at initial presentation and the risk of development of new symptoms ( $p$ value $>0.5$ ). There was statistically significant association between optic nerve hyperintensity and enhancement in MRI orbit and the risk of development of additional neurological deficits in the future $(\mathrm{p}$ value $<0.05$ )

In this study, 2 out of 7 patients with an initial abnormal MRI brain developed new symptoms, but it was not statistically significant $(\mathrm{p}=0.6)$. This was in contrast with findings from ONTT. This discrepancy would have arisen because the study cohort was followed up for a short duration of 1 year and the sample size was also low. If patients with CIS are 
followed up for longer duration, they may meet the criteria for dissemination in time and satisfy criteria for definite MS.

In this study 2 out of the 6 patients with CSF OCB developed new symptoms, but there was no statistically significant $(\mathrm{p}=0.59)$ association. However 2 patients who developed additional deficits satisfied criteria for definite MS. Most studies have shown that individuals with CSF OCB at the time of development of optic neuritis are at higher risk of developing MS [14]. When these patients are followed up for longer duration, the may eventually develop MS. The recent 2017 revisions to the McDonald criteria now allow positive CSF OCB to satisfy dissemination in time criteria. According to this revision, diagnosis of MS can be made with a single clinical event suggestive of demyelination and a single brain MRI. Studies have shown that, in patients satisfying dissemination in space criteria, the presence of CSF OCB positivity increases specificity and has high positive predictive value for the diagnosis of multiple sclerosis.

\section{LIMITATIONS OF THE STUDY}

This study was limited by the number of patients that we were able to recruit for the study and the duration for which they were followed up. So the study may not be sufficiently powered to accurately reflect the temporal evolution and associations of the disease.

\section{CONCLUSION}

In patients presenting with optic neuritis, those with NMO were having the least visual acuity at initial presentation and guarded visual outcome during one year follow up. Retro bulbar neuritis is more common than papillitis in patients with optic neuritis. In patients with bilateral optic neuritis, if S.NMO antibody is negative, it is advisable to test for S. anti MOG antibody. There is statistically significant association between risk of development of multiple sclerosis and increase in the number of lesions during follow up MRI brain. However, there is no significant difference in the risk between those with single versus multiple lesions, in the initial MRI. CSF OCB positivity, if used as dissemination in time criteria in those patients satisfying dissemination in space criteria, helps in the earlier diagnosis of definite MS.

\section{REFERENCES}

1. Foroozan R, Buono LM, Savino PJ, Sergott RC. Acute demyelinating optic neuritis. Current opinion in ophthalmology. 2002 Dec 1; 13(6):375-80.

2. Optic Neuritis Study Group. The clinical profile of optic neuritis: experience of the Optic Neuritis
Treatment Trial. Arch Ophthalmol. 1991; 109:16738.

3. Rizzo JF, Lessell S. Risk of developing multiple sclerosis after uncomplicated optic neuritis A longterm prospective study. Neurology. 1988 Feb 1; 38(2): 185 .

4. Jacobs L, Kinkel PR, Kinkel WR. Silent brain lesions in patients with isolated idiopathic optic neuritis: a clinical and nuclear magnetic resonance imaging study. Archives of neurology. 1986 May 1; 43(5):452-5.

5. Asgari N, Lillevang ST, Skejoe HP, Falah M, Stenager E, Kyvik KO. A population-based study of neuromyelitisoptica in Caucasians. Neurology. 2011 May 3; 76(18):1589-95.

6. Wingerchuk DM, Hogancamp WF, O'brien PC, Weinshenker BG. The clinical course of neuromyelitisoptica (Devic's syndrome). Neurology. 1999 Sep 1; 53(5):1107.

7. Polman $\mathrm{CH}$, Reingold SC, Banwell B, Clanet M, Cohen JA, Filippi M, Fujihara K, Havrdova E, Hutchinson M, Kappos L, Lublin FD. Diagnostic criteria for multiple sclerosis: 2010 revisions to the McDonald criteria. Annals of neurology. 2011 Feb;69(2):292-302.

8. Wingerchuk DM, Banwell B, Bennett JL, Cabre P, Carroll W, Chitnis T, De Seze J, Fujihara K, Greenberg B, Jacob A, Jarius S. International consensus diagnostic criteria for neuromyelitis optica spectrum disorders. Neurology. 2015 Jul 14;85(2):177-89.

9. Saxena R, Phuljhele S, Menon V, Gadaginamath S, Sinha A, Sharma P. Clinical profile and short-term outcomes of optic neuritis patients in India. Indian journal of ophthalmology. 2014 Mar; 62(3):265.

10. Woung LC, Lin CH, Tsai CY, Tsai MT, Jou JR, Chou P. Optic neuritis among national health insurance enrollees in Taiwan, 2000-2004. Neuroepidemiology. 2007; 29(3-4):250-4.

11. Merle H, Olindo S, Jeannin S, Hage R, Donnio A, Richer R, Cabre P. Visual field characteristics in neuromyelitis optica in absence of and after one episode of optic neuritis. Clinical Ophthalmology (Auckland, NZ). 2013;7:1145-1153.

12. Kıyat Atamer A, Ekizoğlu E, Tüzün E, Kürtüncü M, Shugaiv E, Akman Demir G, Eraksoy M. Long term MRI findings in neuromyelitis optica: seropositive versus seronegative patients. European journal of neurology. 2013 May; 20(5):781-7.

13. Beck RW, Trobe JD, Moke PS, Gal RL, Xing D, Bhatti MT, Brodsky MC, Buckley EG, Chrousos GA, Corbett J, Eggenberger E. High-and low-risk profiles for the development of multiple sclerosis within 10 years after optic neuritis: experience of the optic neuritis treatment trial. Archives of ophthalmology (Chicago, Ill.: 1960). 2003 Jul; 121(7):944-9.

14. Tintore M, Rovira A, Rio J, Tur C, Pelayo R, Nos C, Tellez N, Perkal H, Comabella M, Sastre-Garriga J, Montalban X. Do oligoclonal bands add information to MRI in first attacks of multiple sclerosis? Neurology. 2008 Mar 25; 70(13 Part 2):1079-83. 